\title{
Application of 2'-O-(2-N-Methylcarbamoylethyl) Nucleotides in RNase H-Dependent Antisense Oligonucleotides
}

\author{
Yoshiaki Masaki, Yusuke Iriyama, Hiroyuki Nakajima, ${ }^{3}$ Yusuke Kuroda, \\ Tatsuro Kanaki, Satoshi Furukawa, ${ }^{3}$ Mitsuo Sekine, and Kohji Seio ${ }^{1}$
}

An RNase H-dependent antisense oligonucleotide (ASO), having the 2'-O-(2-N-methylcarbamoylethyl) (MCE) modification, was evaluated in vitro and in vivo. The antisense activities of an ASO having the MCE modification were comparable with those of an ASO having the $2^{\prime}$ - $O$-methoxyethyl (MOE) modification in both in vitro and in vivo experiments. In contrast, the hepatotoxic potential of the ASO having the MCE modification was lower than that of the ASO having the MOE modification. Thus, these findings suggested that the MCE modification could be used as an alternative to the MOE modification.

Keywords: RNase-H-dependent antisense, N-Methylcarbamoylethyl (MCE) modification, hepatotoxicity

\section{Introduction}

$\mathbf{R}$ NASE H-DEPENDENT ANTISENSE oligonucleotides (ASOs) are short synthetic oligonucleotides that can modulate the expression of mRNAs of interest. RNase $\mathrm{H}$ is an endonuclease that recognizes the DNA/RNA heteroduplex and cleaves only RNA strands. Thus, RNase H-dependent ASOs need to have a consecutive DNA stretch, often $8-10$ nt in length, to form a DNA/RNA heteroduplex. Chemically modified RNAs are typically added to both the $3^{\prime}$ - and $5^{\prime}$-termini to increase the binding affinity to the target transcript and enhance stability toward nucleases. This construct is called the "gapmer."

Chemical modification of the gapmer has been extensively studied [1], and the 2'-O-methoxyethyl (MOE) modification is one of the most prominent examples. Introduction of MOE to ASOs increases their binding affinity toward target transcripts and promotes resistance to nucleases. This modification is currently used in mipomersen, a US Food and Drug Administration-approved RNase H-dependent antisense drug. ASOs containing locked nucleic acids (LNAs)/bridged nucleic acids (BNAs) show significantly improved binding affinities and nuclease resistances. These physical and biochemical properties enable the concentration of ASOs to be reduced [2-4].
However, introduction of some chemical modifications can increase the risk of toxicity in vivo [5]. Indeed, incorporation of LNAs with particular sequences induces hepatotoxic effects, whereas incorporation of MOE-modified nucleotides in the same sequence does not cause toxicity. This strong hepatotoxic effect can be avoided by using constrained ethyl (cEt) nucleoside [3], N-MeO-amino BNA, NMe-aminooxy BNA, or 2', 4'-BNA ${ }^{\mathrm{NC}}[\mathrm{NMe}$ ] [4]. However, even in the case of mipomersen, a MOE-modified ASO, $10 \%-20 \%$ of patients exhibit a risk of liver damages. Thus, suppression of toxicity is an important factor for developing chemically modified ASOs.

We have recently reported the synthesis and properties of 2'-O-methylcarbamoyl ethyl (MCE)-modified nucleotides (Fig. 1). These modified nucleosides can be easily synthesized through the oxa-Michael reaction [6]. In addition, this modification can improve resistance to nucleases, and the binding affinity to target transcript is comparable to that of 2'-O-methyl modification [7]. Interestingly, in our preliminary experiments of exon skipping therapy, an RNase $\mathrm{H}$-independent antisense mechanism, incorporation of only six MCE nucleosides into 2'-O-methyl-modified ASOs, was found to significantly increase efficacy [6]. However, its applications in RNase Hdependent ASOs and the toxicological properties of the generated ASOs are still unknown.

\footnotetext{
${ }^{1}$ Department of Life Science and Technology, Tokyo Institute of Technology, Yokohama, Japan.

${ }^{2}$ Nissan Chemical Corporation, Chemical Research Laboratories, Funabashi, Japan.

${ }^{3}$ Nissan Chemical Corporation, Biological Research Laboratories, Shiraoka, Japan.
}

(C) Yoshiaki Masaki et al. 2018; Published by Mary Ann Liebert, Inc. This Open Access article is distributed under the terms of the Creative Commons Attribution Noncommercial License (http://creativecommons.org/licenses/by-nc/4.0/) which permits any noncommercial use, distribution, and reproduction in any medium, provided the original author(s) and the source are cited. 


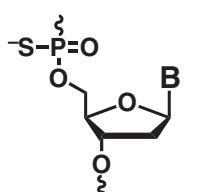

DNA

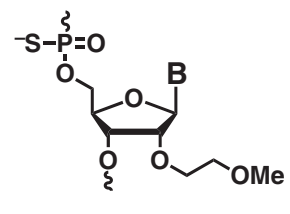

2'-O-MOE nucleotides

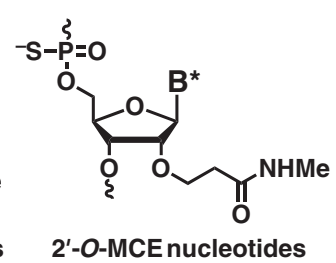

FIG. 1. Chemical structures of modified nucleotides used in this study. B represents A, T, G, and $\mathrm{m}^{5} \mathrm{C}$. B* represents A, $\mathrm{U}, \mathrm{G}$, and $\mathrm{C}$.

Accordingly, in this study, we investigated the applications of MCE-modified ASOs and the influence of the modification on hepatotoxic effects.

\section{Materials and Methods}

\section{Oligonucleotides}

A series of ASOs were synthesized using an nS8-II automated DNA Synthesizer (Gene Design) and purified. The sequences of the ASOs targeting phosphatase and tensin homolog (PTEN) mRNA were as follows: MCE ASO, PS-d $\left(\mathrm{C}_{M C E} \mathrm{U}_{M C E} \mathrm{G}_{M C E} \mathrm{C}_{M C E} \mathrm{U}_{M C E} A G^{\mathrm{m}} \mathrm{C}^{\mathrm{m}} \mathrm{CT}^{\mathrm{m}} \mathrm{CTGGAU}_{M C E} \mathrm{U}_{M C E}\right.$ $\left.\mathrm{U}_{M C E} \mathrm{G}_{M C E} \mathrm{~A}_{M C E}\right)$; and MOE ASO, PS-d $\left({ }^{\mathrm{m}} \mathrm{C}_{\mathrm{MOE}} \mathrm{T}_{\mathrm{MOE}} \mathrm{G}_{\mathrm{MOE}}{ }^{\mathrm{m}}\right.$ $\left.\mathrm{C}_{\mathrm{MOE}} \mathrm{T}_{\mathrm{MOE}} \mathrm{AG}^{\mathrm{m}} \mathrm{C}^{\mathrm{m}} \mathrm{CT}^{\mathrm{m}} \mathrm{CTGGAT}_{\mathrm{MOE}} \mathrm{T}_{\mathrm{MOE}} \mathrm{T}_{\mathrm{MOE}} \mathrm{G}_{\mathrm{MOE}} \mathrm{A}_{\mathrm{MOE}}\right)$.

\section{In vitro knockdown experiments}

The bEnd. 3 cell line was obtained from the American Type Culture Collection. bEnd. 3 cells were grown in $100 \mathrm{~mm} \times 20$ $\mathrm{mm}$ Style Dishes incubated at $37^{\circ} \mathrm{C}$ in the presence of $10 \% \mathrm{CO}_{2}$ in complete growth medium (Dulbecco's modified Eagle's medium supplemented with 5\% fetal bovine serum; Wako Pure Chemical Industries, Ltd., Osaka, Japan; Corning, Inc., Corning, NY) until they reached $70 \%-80 \%$ confluence. Once confluence was reached, the cells were either subcultured or plated at $2 \times 10^{3}$ cells/well into 96-well plates for transfection the following day. On the day of transfection, ASOs and small interfering RNA (siRNA) were transfected at final concentrations of 10 or $100 \mathrm{nM}$ using RNAiMAX (Thermo Fisher Scientific) or Oligofectamine (Thermo Fisher Scientific) according to the manufacturer's protocol. The plates were incubated at $37^{\circ} \mathrm{C}$ for $4 \mathrm{~h}$ in the presence of $5 \% \mathrm{CO}_{2}$. Then the transfection mixtures were removed, and the cells were refed with complete medium. After $20 \mathrm{~h}$ of incubation, the cells were washed once with $1 \times$ phosphate-buffered saline and lysed, and total mRNA was isolated using Qiagen 96-well RNeasy plates (Qiagen, Valencia, CA). The isolated RNA was analyzed for PTEN and cyclophilin A mRNA expression levels using quantitative real-time reverse transcription-polymerase chain reaction (qRT-PCR). The primer probes for mouse PTEN and cyclophilin A were Mm00477208_m1 and Mm0234230_g1 (all from Thermo Fisher Scientific), respectively. Analysis was done by the $\triangle \triangle \mathrm{Ct}$ threshold method. PTEN mRNA expression levels for each well were normalized to those of cyclophillin A and expressed as a percentage of the levels observed in untreated control wells. Each treatment was performed in triplicate on separate 96-well plates.

\section{In vivo animal experiments}

These experiments were conducted according to the Guidelines for Animal Experimentation, Japanese Associa- tion for Laboratory Animal Science. Male BALB/c mice (6 weeks old; Charles River Laboratories, Japan, Inc.) were housed at one animal per cage. For the multiple administration studies, ASOs were administered in physiological saline by intraperitoneal (i.p.) injection at the indicated dose, twice a week for 3 weeks. The animals were maintained at a constant temperature of $22^{\circ} \mathrm{C}$ and were fed a standard laboratory diet. Animal weight was recorded before each dosing throughout the live phase of the study. Mice were anesthetized and sacrificed 3 days after administration of the final dose. Plasma was isolated from whole blood collected from the abdominal aorta under anesthesia. Alanine aminotransferase (ALT), aspartate aminotransferase (AST), and lactate dehydrogenase (LDH) levels were determined using an automated clinical chemistry analyzer (Fuji-DRI-CHEM 7000; FUJIFILM Medical Co., Ltd.). Organs, including the liver, skeletal muscle, and brain, were collected and homogenized in ISOGEN II (NIPPON GENE Co., Ltd.). RNA was extracted using RNeasy columns (Qiagen) according to the manufacturer's protocol. RNA was eluted from the columns with water. mRNA samples were reverse transcribed to template DNA using Primescript ${ }^{\mathrm{TM}}$ RT Master Mix (Perfect Real Time, Ta$\mathrm{KaRa}$ ) according to the manufacturer's protocol, and GeneAmp ${ }^{\circledR}$ PCR System 9700 (Applied Biosystems, Foster City, CA). Template DNA samples were analyzed by fluorescencebased qRT-PCR using Premix Ex Taq ${ }^{\mathrm{TM}}$ (Perfect Real Time) according to the manufacturer's protocol, and an Applied Biosystems 7700 sequence detector (Applied Biosystems). The primer probes for mouse PTEN and cyclophilin A were Mm00477208_m1 and Mm0234230_g1 (all from Thermo Fisher Scientific), respectively. Analysis was done by the $\Delta \Delta \mathrm{Ct}$ threshold method. Levels of target mRNAs and cyclophilin A were evaluated. Target mRNA levels were normalized to cyclophilin levels for each sample.

\section{UV melting analysis}

Each oligonucleotide was dissolved in $10 \mathrm{mM}$ sodium phosphate buffer ( $\mathrm{pH} 7.0$ ), $100 \mathrm{mM} \mathrm{NaCl}, 0.1 \mathrm{mM}$ EDTA to arrange the final concentration of each oligonucleotide to be $2.0 \mu \mathrm{M}$. The solution was separated into quarts cells $(10 \mathrm{~mm})$ and incubated at $95^{\circ} \mathrm{C}$. After $10 \mathrm{~min}$, the solution was cooled to $5^{\circ} \mathrm{C}$ at a rate of $0.5^{\circ} \mathrm{C} / \mathrm{min}$. Then, the solution was kept at $5^{\circ} \mathrm{C}$ for $5 \mathrm{~min}$. After $5 \mathrm{~min}$, the solution was heated at $95^{\circ} \mathrm{C}$ at the same rate. The absorption at $260 \mathrm{~nm}$ was recorded and used to draw UV-melting curves. The obtained melting curves were smoothed using Savitzky-Golay treatment. $T_{\mathrm{m}}$ was calculated as the temperature that gave the maximum of the first derivative of the UV-melting curve.

\section{Statistics}

Mean values were used as a representative value. Error bars represent standard deviations. The mean values were compared using Tukey tests. Differences with $P$ values of less than 0.05 were considered statistically significant.

\section{Results}

\section{MCE and MOE modifications showed similar RNase $\mathrm{H}$-dependent antisense activity in vitro}

To examine the effects of the MCE modification on antisense activity, in vitro mRNA knockdown experiments were 

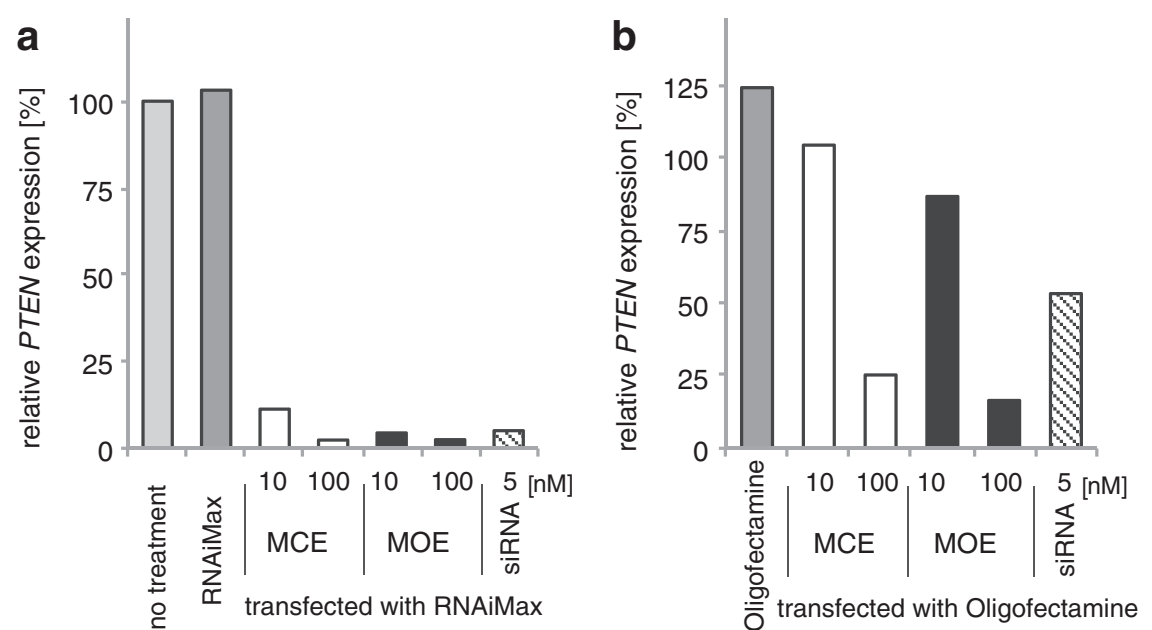

FIG. 2. Antisense activities of chemically modified ASOs in vitro. Relative PTEN expression was calculated using the following formula: (PTEN expression level in treated cells $) /(P T E N$ expression level in untreated cells). ASO, antisense oligonucleotide. (a) ASOs were transfected using RNAiMax. (b) ASOs were transfected with Oligofectamine. performed. The synthesized ASOs targeted PTEN mRNA, and the sequence was the same as that reported by Swayze et al. [5]. As a positive control, MOE-modified ASOs and siRNA were used, and as a negative control, no treatment or treatment with the transfection reagent only was performed in bEnd.3 mouse brain endothelial cells. The antisense activities are shown in Fig. 2. The relative PTEN expression level in cells transfected with $10 \mathrm{nM}$ MCE-modified ASOs using RNAiMax reagent was $11 \%$, and that in cells transfected with $100 \mathrm{nM}$ MCE-modified ASOs was $2.4 \%$. Similarly, those of 10 and $100 \mathrm{nM}$ MOE-modified ASOs were $4.4 \%$ and $2.5 \%$, respectively. When using Oligofectamine as a transfection reagent, the relative $P T E N$ expression levels in cells transfected with 10 and $100 \mathrm{nM}$ MCE-modified ASOs were $104 \%$ and $25 \%$, respectively. Similarly, those in cells transfected with 10 and $100 \mathrm{nM}$ MOE-modified ASOs were $87 \%$ and $16 \%$, respectively. In both experiments, we observed similar concentration-dependent reductions in mRNA expression. These data suggested that MCE-modified ASOs showed similar antisense activity as MOE-modified ASOs.

\section{Comparison of the effects of MCE and MOE modifications on antisense activity}

The relative PTEN mRNA expression levels in mice treated with MCE-modified ASOs at doses of $3.2 \mu \mathrm{mol} / \mathrm{kg}$ $(23.6 \mathrm{mg} / \mathrm{kg})$ and $6.4 \mu \mathrm{mol} / \mathrm{kg}(47.2 \mathrm{mg} / \mathrm{kg})$ in the liver were $58 \% \pm 7.3 \%$ and $44 \% \pm 6.6 \%$, respectively (Fig. 3 , left panel). The PTEN mRNA expression levels were reduced in a dosedependent manner. Similarly, those in mice treated with
MOE-modified ASOs at doses of $3.2 \mu \mathrm{mol} / \mathrm{kg}(23.1 \mathrm{mg} / \mathrm{kg})$ and $6.4 \mu \mathrm{mol} / \mathrm{kg}(46.2 \mathrm{mg} / \mathrm{kg})$ in the liver were $55 \% \pm 12 \%$ and $34 \% \pm 8.2 \%$. The antisense activities of MCE-modified ASOs in the liver were comparable to those of MOEmodified ASOs. In the muscle, the relative PTEN mRNA expression levels in mice treated with MCE-modified ASOs at doses of 3.2 and $6.4 \mu \mathrm{mol} / \mathrm{kg}$ were $75 \% \pm 18 \%$ and $68 \% \pm 19 \%$, respectively (Fig. 3, middle panel). These values were similar to those observed for MOE-modified ASOs $(75 \% \pm 24 \%$ and $68 \% \pm 21 \%$, respectively). The mean mRNA expression levels showed dose-dependent changes, but statistical significance was not observed. In the brain, the relative PTEN mRNA expression levels in mice treated with MCE-modified ASOs at doses of 3.2 and $6.4 \mu \mathrm{mol} / \mathrm{kg}$ were $83 \% \pm 22 \%$ and $84 \% \pm 25 \%$, respectively (Fig. 3, right panel). Similarly, those in mice treated with MOE-modified ASOs were $70 \% \pm 8.4 \%$ and $95 \% \pm 44 \%$, respectively. Dosedependent reductions in mRNA levels were not observed in both experiments using MCE- or MOE-modified ASOs. Thus, these data showed that i.p. administration of MCEmodified ASOs resulted in biodistributions and antisense activities similar to those of MOE-modified ASOs in vivo.

\section{MCE modification reduced hepatotoxic potential}

To assess the safety of chemically modified ASOs, liver function was evaluated. (Supplementary Tables S1-S4; Supplementary Data are available online at www.liebertpub .com/nat). Previous reports have shown that a dose of $2.25 \mu \mathrm{mol} / \mathrm{kg}$ of the same sequence of LNAs, that is, 2-16-2
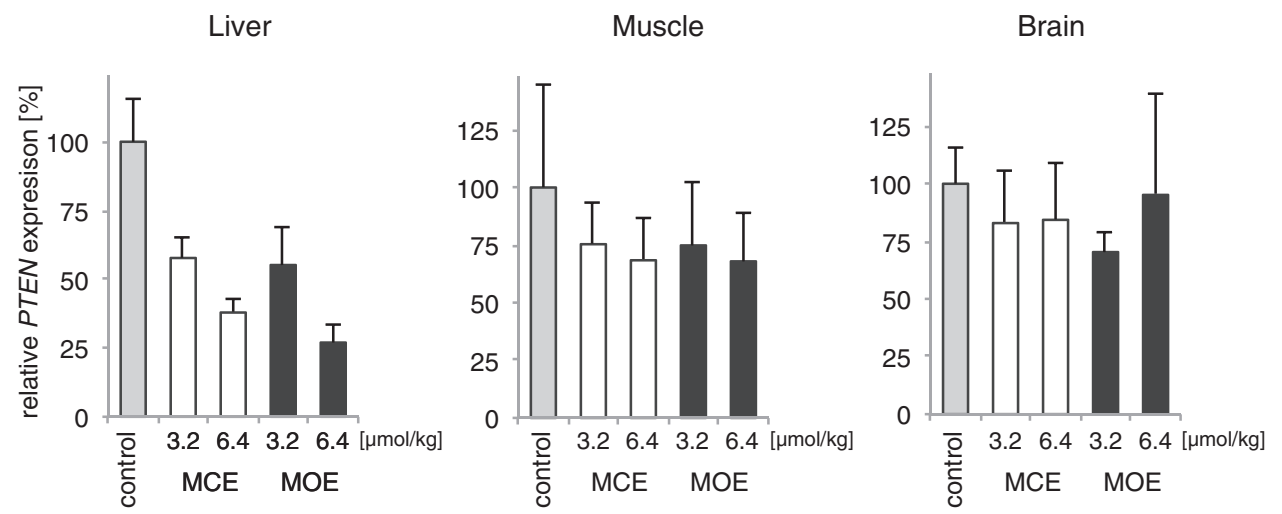

FIG. 3. PTEN mRNA expression in the liver, muscle, and brain. Naked ASOs were administrated intraperitoneally to mice (6-week-old male $\mathrm{BALB} / \mathrm{c}$ mice, $n=4)$ twice a week for 3 weeks. The mice were scarified 3 days after the last administration, and mRNA levels in the liver, muscle, and brain were quantified. Error bars indicate standard deviations. 
FIG. 4. AST, ALT, and LDH levels after the 3 -week multiple administration study. All data were obtained from the same mice used in Figure 3. $* P<0.05$, Tukey's test. ALT, alanine aminotransferase; AST, aspartate aminotransferase; LDH, lactate dehydrogenase. (a) AST levels. (b) ALT levels. (c) LDH levels.
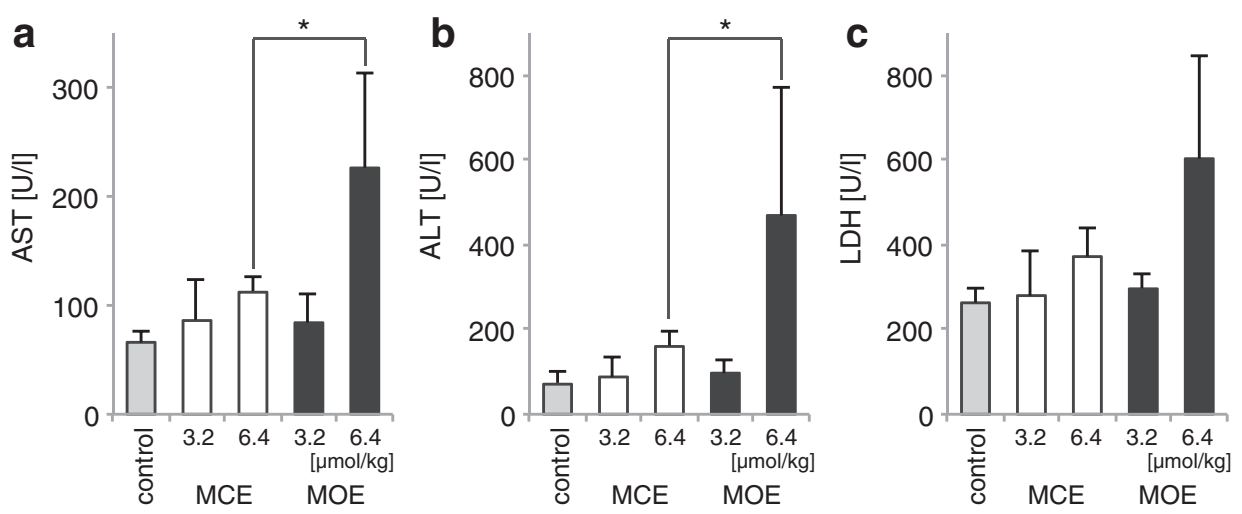

Gapmer, induces significant hepatotoxicity in mice [5]. In contrast, MOE-modified ASOs were well tolerated in the $2.25 \mu \mathrm{mol} / \mathrm{kg}$ dose group. In our study, the levels of AST, ALT, and LDH in the $3.2 \mu \mathrm{mol} / \mathrm{kg} \mathrm{MCE}$-modified ASO dose group were $86 \pm 38,88 \pm 48$, and $280 \pm 106 \mathrm{U} / \mathrm{L}$, respectively (Fig. 4). These values in the $6.4 \mu \mathrm{mol} / \mathrm{kg}$ MCE-modified ASO dose group were $112 \pm 15,160 \pm 36$, and $372 \pm 66 \mathrm{U} / \mathrm{L}$, respectively. For MOE-modified ASOs, the levels of AST, ALT, and $\mathrm{LDH}$ in the $3.2 \mu \mathrm{mol} / \mathrm{kg}$ dose group were $84 \pm 26,97 \pm 32$, and $296 \pm 34 \mathrm{U} / \mathrm{L}$, respectively, and those in the $6.4 \mu \mathrm{mol} / \mathrm{kg}$ dose group were 226 $\pm 88,469 \pm 302$, and $603 \pm$ $246 \mathrm{U} / \mathrm{L}$, respectively. The differences in AST and ALT levels induced by $6.4 \mu \mathrm{mol} / \mathrm{kg}$ MCE- or MOE-modified ASOs were statistically significant. Taken together, our results suggested that MCE-modified ASOs could be used as alternatives to MOE-modified ASOs to increase the safety of RNase $\mathrm{H}-$ dependent ASOs.

\section{Discussion}

In this study, we found that RNase H-dependent ASOs with the MCE modification were less hepatotoxic than those with the MOE modification, although the on-target effects of the two ASOs were comparable. It should be noted that MOE-modified ASO/RNA duplex showed higher melting temperature $\left(72^{\circ} \mathrm{C} \pm 0.3^{\circ} \mathrm{C}\right)$ than MCE-modified ASO/RNA duplex $\left(68^{\circ} \mathrm{C} \pm 0.2^{\circ} \mathrm{C}\right)$ Supplementary Fig. S1. This is because MOE-modified nucleosides have 5-methyl group on the pyrimidine bases (seven positions in total), whereas MCE-modified nucleosides do not have. In addition, it is reported that MOE-modified RNA/RNA duplex have higher duplex stability than 2'-O-methyl RNA/RNA duplex, whereas MCE-modified RNA/RNA duplex have similar duplex stability with 2'-O-methyl RNA/RNA duplex [7-9]. These structural differences may contribute to the difference of their duplex stabilities.

The mechanisms of hepatotoxicity can be categorized into two classes: hybridization-dependent off-target effects and hybridization-independent effects. The mechanisms of hybridization-dependent off-target effects are mainly explained by RNase H-dependent knockdown of unintended transcripts [10-13]. It is reported that high affinity of gapmer oligonucleotides to target RNA are closely related to the high hepatotoxic potential. This relationship could be explained by RNase H-dependent knockdown of unintended transcripts [13]. Because MCE-modified ASO has lower duplex stability than MOE-modified ASO, it may be possible to explain that MCE-modified ASO-mediated RNase H-dependent knockdown of unintended transcripts was less significant than the MOE-modified ASO-mediated one.

On the other hand, because the on-target effects of ASOs used in this study were similar, RNase H-dependent knockdown of unintended transcripts could be also assumed to have similar profiles for both ASOs. Thus, it may be possible to explain the differences in hepatotoxicity between MCE and MOE modifications by hybridization-independent effects. One of the major mechanisms mediating the hybridization-independent effects is the binding of chemically modified ASOs to plasma and cellular proteins. Introduction of chemical modifications on ASOs is known to result in differential binding affinities to cellular proteins $[14,15]$. For example, 2'-F-modified oligonucleotides cause rapid degradation of $\mathrm{P} 54 \mathrm{nrb}$ and polypyrimidine tractbinding protein-associated splicing factor (PSF) which belong to the Drosophila behavior/human splicing family [16]. Although the detailed mechanisms are unknown, 2'-F-modified ASOs may directly interact with these proteins, resulting in impairment of cell proliferation. Thus, suppression of unwanted protein binding could be a potential approach to reduce unexpected toxicity. The retention time of MCE-modified ASO in reverse phase high performance liquid chromatography (HPLC) was $11.7 \mathrm{~min}$, whereas that of MOE-modified ASO was 14.1 min (Supplementary Fig. S2). This difference suggests that MCE-modified ASO is more hydrophilic than MOE-modified ASO. It is said that more hydrophobic modification at the $2^{\prime}$ moiety of the ASO tends to bind to proteins promiscuously, and show greater affinity with the proteins [14]. Thus, the hydrophilic nature of MCE modification could suppress unwanted protein binding, resulting in reduced hepatotoxicity. It is also possible that hydrophilic nature changes plasma protein binding of ASO, which will change the pharmacokinetic nature of the ASO. Further study will be needed to understand the effect of MCE modification.

In summary, we investigated the applications of MCEmodified ASOs and the influence of these ASOs on hepatotoxic effects. MCE-modified ASO showed on-target effects similar to those of MOE-modified ASOs in both in vitro and in vivo experiments. In contrast, liver blood tests indicated that MCE-modified ASOs showed increased safety compared with MOE-modified ASOs. Our results suggested that MCEmodified ASOs could be used as an alternative to MOEmodified ASOs to increase the safety of RNase H-dependent ASOs. 


\section{Acknowledgment}

This study was supported by JSPS KAKENHI (grant nos. 17H04886 and 26810086).

\section{Author Disclosure Statement}

No competing financial interests exist.

\section{References}

1. Khvorova A and JK Watts. (2017). The chemical evolution of oligonucleotide therapies of clinical utility. Nat Biotechnol 35:238-248.

2. Straarup EM, N Fisker, M Hedtjarn, MW Lindholm, C Rosenbohm, V Aarup, HF Hansen, H Orum, JBR Hansen and T Koch. (2010). Short locked nucleic acid antisense oligonucleotides potently reduce apolipoprotein B mRNA and serum cholesterol in mice and non-human primates. Nucleic Acids Res 38:7100-7111.

3. Seth PP, A Siwkowski, CR Allerson, G Vasquez, S Lee, TP Prakash, EV Wancewicz, D Witchell and EE Swayze. (2009). Short antisense oligonucleotides with novel 2'-4' conformationaly restricted nucleoside analogues show improved potency without increased toxicity in animals. J Med Chem 52:10-13.

4. Prakash TP, A Siwkowski, CR Allerson, MT Migawa, S Lee, HJ Gaus, C Black, PP Seth, EE Swayze and B Bhat. (2010). Antisense oligonucleotides containing conformationally constrained $2^{\prime}, 4^{\prime}$-(N-methoxy)aminomethylene and $2^{\prime}, 4^{\prime}$ aminooxymethylene and $2^{\prime}-\mathrm{O}, 4^{\prime}$-C-aminomethylene bridged nucleoside analogues show improved potency in animal models. J Med Chem 53:1636-1650.

5. Swayze EE, AM Siwkowski, EV Wancewicz, MT Migawa, TK Wyrzykiewicz, G Hung, BP Monia and CF Bennett. (2007). Antisense oligonucleotides containing locked nucleic acid improve potency but cause significant hepatotoxicity in animals. Nucleic Acids Res 35:687-700.

6. Yamada T, N Okaniwa, H Saneyoshi, A Ohkubo, K Seio, T Nagata, Y Aoki, S Takeda and M Sekine. (2011). Synthesis of 2'-O-[2-(N-methylcarbamoyl)ethyl]ribonucleosides using oxa-Michael reaction and chemical and biological properties of oligonucleotide derivatives incorporating these modified ribonucleosides. J Org Chem 76:3042-3053.

7. Yamada T, Y Masaki, N Okaniwa, T Kanamori, A Ohkubo, H Tsunoda, K Seio and M Sekine. (2014). Synthesis and properties of oligonucleotides modified with 2'-O-(2carboxyethyl)nucleotides and their carbamoyl derivatives. Org Biomol Chem 12:6457-6464.

8. Altmann KH, NM Dean, D Fabbro, SM Freier, T Geiger, R Häner, D Hüsken, P Martin, BP Monia, et al. (1996). Second generation of antisense oligonucleotides: from nu- clease resistance to biological efficacy in animals. Chimia 53:168-176.

9. Masaki Y, R Miyasaka, A Ohkubo, K Seio and M Sekine. (2010). Linear relationship between deformability and thermal stability of 2'-O-modified RNA hetero duplexes. J Phys Chem B 114:2517-2524.

10. Burel SA, CE Hart, P Cauntay, J Hsiao, T Machemer, M Katz, A Watt, HH Bui, H Younis, et al. (2016). Hepatotoxicity of high affinity gapmer antisense oligonucleotides is mediated by RNase $\mathrm{H} 1$ dependent promiscuous reduction of very long pre-mRNA transcripts. Nucleic Acids Res 44: 2093-2109.

11. Kasuya T, S Hori, A Watanabe, M Nakajima, Y Gahara, M Rokushima, T Yanagimoto and A Kugimiya. (2016). Ribonuclease H1-dependent hepatotoxicity caused by locked nucleic acid-modified gapmer antisense oligonucleotides. Sci Rep 6:30377.

12. Kamola PJ, JDA Kitson, G Turner, K Maratou, S Eriksson, A Panjwani, LC Warnock, GAD Guilloux, K Moores, et al. (2015). In silico and in vitro evaluation of exonic and intronic off-target effects form a critical element of therapeutic ASO gapmer optimization. Nucleic Acids Res 43:8638-8650.

13. Dieckmann A, PH Hagedorn, Y Burki, C Brugmann, M Berrera, M Ebeling, T Singer and F Schuler. (2018). A sensitive in vitro approach to assess the hybridizationdependent toxic potential of high affinity gapmer oligonucleotides. Mol Ther Nucleic Acids 10:45-54.

14. Crooke ST, SY Wang, TA Vickers, W Shen and XH Liang. (2017). Cellular uptake and trafficking of antisense oligonucleotides. Nat Biotechnol 35:230-237.

15. Bailey JK, W Shen, XH Liang and ST Crooke. (2017). Nucleic acid binding proteins affect the subcellular distribution of phosphorothioate antisense oligonucleotides. Nucleic Acids Res 45:10649-10671.

16. Shen W, XH Liang, H Sun and ST Crooke. (2015). 2'Fluoro-modified phosphorothioate oligonucleotide can cause rapid degradation of P54nrb and PSF. Nucleic Acids Res 43:4569-4578.

Address correspondence to:

Kohji Seio, $P h D$

Department of Life Science and Technology

Tokyo Institute of Technology 4259 Nagatsuta Midoriku Yokohama 226-8501

Japan

E-mail: kseio@bio.titech.ac.jp

Received for publication May 16, 2018; accepted after revision June 12, 2018. 